\title{
SISTEMA DE INDICADORES SOCIALES SOBRE LA MUJER *
}

\author{
M. Luisa Moltó \\ (Universidad de Valencia)
}

\section{INTRODUCCION}

La insatisfacción derivada de la utilización de indicadores económicos convencionales para medir el nivel de bienestar ha tenido como consecuencia el desarrollo de indicadores sociales que evalúen directamente el bienestar de las personas, tanto en términos monetarios como no monetarios. Planteada la necesidad de contemplar los aspectos no monetarios de la economía, al final de la década de los sesenta aparecen dos tendencias. La primera consiste en valorat monetariamente estos aspectos no monetarios que contribuyen al crecimiento del bienestar, como puede ser la producción de bienes y servicios de las amas de casa, incluyéndolos, por tanto, en las macromagnitudes que conforman la contabilidad nacional. La segunda tiene como objetivo captar la tendencia y los aspectos cualitativos que reflejan las condiciones sociales.

En cualquier análisis de la situación de las mujeres se utilizan actualmente indicadores que permiten realizar comparaciones, por una parte, con respecto a los varones, si de lo que se trata es de cuantificar las desigualdades; $y$, por otro lado, en el tiempo y el espacio, si de lo que se trata es de obtener tendencias o evaluar los resultados de determinadas políticas públicas. Si bien los indicadores tienen como objetivo inmediato la descripción, su utilidad en último término es la evaluación de la efectividad de las políticas gubernamentales. Véase Ruggles \& Ruggles (1974).

* Aquest article va aparèixer a «Información Comercial Española»/27, març 1988. 
Es conveniente, sin embargo, que los indicadores se encuentren enmarcados en un sistema con el fin de asegurar que están captando, al menos, las principales dimensiones del problema de estudio.

El propósito de este trabajo es, en primer lugar, describir brevemente en qué consiste un sistema de indicadores sociales. En segundo lugar, desarrollar el proyecto de elaboración de un modelo de indicadores sociales sobre la situación de la mujer en los dos últimos años, y, finalmente, describir someramente la situación de la mujer en España en base a un conjunto de indicadores.

\section{SISTEMA DE INDICADORES SOCIALES}

Los indicadores sociales, según Horn (1980), constituyen medidas cuantitativas de los fenómenos sociales, aplicándose al estudio de las relaciones sociales en diferentes ámbitos: familia, educación, salud, empleo, etc.

Para reflejar cuantitativamente los aspectos generales de la vida económica y social de las mujeres es necesario realizar previamente un análisis cualitativo de las variables que se consideran fundamentales y establecer sus principales relaciones.

La obtención de una medida del bienestar de las mujeres puede ser posible actualmente sin mucha dificultad mediante los indicadores sociales, que no sólo ponen de manifiesto las condiciones sociales de las mujeres, sino que en el mismo proceso de medición contribuyen a conceptualizar dichas condiciones sociales. Los indicadores sociales se pueden considerar a la vez instrumentos de medida y de definición de lo que se desea medir. Su desarrollo implica el establecimiento de un proceso de aprendizaje continuado, en el seno del cual se irán redefiniendo las categorías básicas que permitan analizar la situación pasada, presente y futura de las mujeres y sus relaciones con los varones.

Las estadísticas disponibles constituyen la base necesaria para la elaboración de indicadores sociales. Sin embargo, la diferencia entre un indicador y las estadísticas estriba en que estas últimas reflejan un conjunto de hechos numéricos recopilados de forma sistemática, mientras que para obtener un indicador es necesario condensar la información contenida en las estadísticas existentes en relación con la idea o concepto básico que se trata de medir, con la finalidad de establecer comparaciones, tanto en el tiempo como en el espacio.

Por otra parte, se sabe que las tendencias en lo social y en lo económico no se encuentran completamente determinadas en el futuro, y por 
tanto existen posibilidades de que se produzca una evolución más o menos favorable en relación con un objetivo prefijado. Estas posibilidades se encuentran condicionadas por la incertidumbre asociada a este tipo de fenómenos. Pero dicha incertidumbre se puede disminuir si se conocen las restricciones, tanto de tipo técnico como social, que delimitan los cambios considerados factibles, dentro de una tecnología y unos límites espaciales y demográficos determinados.

Se trata, en definitiva, de establecer diagnósticos respecto a la dirección del cambio de las desigualdades de las mujeres con respecto a los varones en todos los ámbitos.

\section{EL MODELO DE INDICADORES SOCIALES SOBRE LA SITUACION DE LA MUJER DEL INSTITUTO DE LA MUJER}

El proyecto de elaboración de un sistema de indicadores sociales sobre la situación de la mujer, auspiciado por el Instituto de la Mujer, se desarrolla en tres fases. En la primera se definen las principales áreas en las cuales se puede dividir, en una primera aproximación, el conocimiento de la situación de la mujer; se estudia las dimensiones teóricas y sus contrapartidas prácticas en cada una de las áreas $\mathrm{y}$, finalmente, se debate la adopción del sistema de indicadores propuesto por las Naciones Unidas.

Las razones por las cuales se adopta el sistema de las Naciones Unidas, expuestas en Astelarra (1986), son las siguientes: Por un lado, el modelo de las Naciones Unidas es el más sistemático y completo, internacionalmente, proponiendo un conjunto de indicadores específicos en cada una de las áreas de preocupación social. Por otra parte, y teniendo en cuenta que dicho modelo se establece a partir de las estadísticas existentes en cada país, su puesta en práctica en España permite valorar la calidad de las estadísticas para medir la situación de la mujer. Así, las deficiencias estadísticas observadas pueden servir de base para su mejora, realizando propuestas a los organismos competentes, que en la mayor parte de los casos implican únicamente leves modificaciones en la presentación de los datos. Tal es el caso de la desagregación por sexo de la mayor parte de los datos disponibles. Además, la sencillez del modelo de las Naciones Unidas permite

1. Este sistema de indicadores ha sido elaborado por el International Research and Training Institute for the Advancement of Women (INSTRAW), organismo dependiente de las Naciones Unidas, especializado en temas acerca de la mujer. Para una información más detallada, pueden verse los informes de la ONU (1984) y el trabajo de Torns y Carrasquer (1985). 
organizar un sistema de soporte de datos sobre indicadores y fuentes estadísticas.

Sin embargo, la contrapartida a la sencillez de este modelo y su amplia aplicación en el ámbito internacional es que el conjunto de indicadores propuesto es excesivamente simple y elemental. Por tanto, tiene una capacidad limitada para reflejar la situación de las mujeres en los países más desarrollados donde los mecanismos de discriminación adoptan formas cada vez más sutiles, porque se requieren indicadores más elaborados para aprehenderla.

A pesar de las deficiencias que este sistema de indicadores presenta, especialmente en algunas de las áreas, se decidió finalmente adoptarlo como base de partida para confrontarlo con el conjunto de las estadísticas generales y específicas disponibles en la actualidad.

En la segunda fase se obtiene un fichero de indicadores sociales sobre la situación de la mujer.

La elaboración de fichas de los indicadores sociales se realiza, en una primera instancia, a partir de los indicadores de las Naciones Unidas y de unas listas de indicadores adicionales propuestas en la primera etapa de la investigación. El propósito de estas listas de indicadores adicionales es recoger aquellas peculiaridades de la situación española, difícilmente reflejadas por los indicadores de las Naciones Unidas, completando, pues, los vacíos no cubiertos en algunas de las áreas (trabajo, educación y salud).

El modelo de ficha de indicador contiene las especificaciones imprescindibles para la recopilación de la información básica acerca de los datos necesarios para elaborar el indicador, de forma sistematizada, con el fin de facilitar al máximo la tecogida de datos para la obtención de series históricas. Es interesante también hacer constar en las observaciones todo tipo de información que contribuya a clarificar las posibilidades de su utilización en otros ámbitos espaciales, y para el establecimiento de comparaciones internacionales. A este respecto son importantes tanto el nivel de desagregación de los datos, como la definición precisa de las variables que se encuentran involucradas en el cálculo del indicador.

Por otro lado, el modelo de ficha de fuentes estadísticas debe ser utilizado cuando la fuente estadística sirva para la elaboración de diversos indicadores, o en el caso de que contenga información relevante o bien de variables clasificadas por sexo. En este último caso se podrían extraer sugerencias para nuevas propuestas de indicadores.

A grandes rasgos, se pueden distinguir dos tipos de fuente. La estadística propiamente dicha y las elaboraciones de la estadística, que generalmente se presentan en forma de publicaciones.

Es importante tomar en consideración que la obtención de la informa- 
ción para la elaboración de series históricas sobre los indicadores sociales no requiere necesariamente acudir a la estadística propiamente dicha. Las publicaciones, sobre todo recientemente, contienen elaboraciones de los datos muy ricas en información. Además, en muchos casos el indicador deseado no supone únicamente la obtención de un valor, sino que hace referencia a una tabla de valores, en muchas ocasiones publicada con un formato adecuado a las necesidades. Por tanto, la tendencia general debe ser acudir directamente a las publicaciones, si bien es conveniente tener presente que las publicaciones no son homogéneas entre sí, ni tampoco lo son consigo mismas a lo largo del tiempo debido a cambios en la metodología empleada. Si el objetivo fuera la construcción de las series de cara al futuro, ello no plantearía excesivos problemas. Sin embargo, puesto que interesa conocer los antecedentes de la situación actual, los problemas pueden ser graves cuando se intenta ir hacia el pasado.

Así, pues, en la elaboración del fichero de fuentes estadísticas se utilizan mayoritariamente las publicaciones, y sólo en casos muy determinados se pone de manifiesto la necesidad de acudir a la estadística propiamente dicha, que contiene más información, pero que implica conocer la estructura del fichero informático.

$\mathrm{Si}$ el objetivo prioritario de un indicador es establecer comparaciones internacionales, es conveniente utilizar fuentes internacionales, ya que si bien estos organismos recaban la información de instituciones y organizaciones nacionales, y pocas veces realizan directamente la estadística, el proceso de elaboración de los datos suministrados por los diversos países garantiza que las comparaciones sean significativas.

Posteriormente, tras un debate con investigadoras e investigadores especializados en las diversas áreas sobre las fichas elaboradas provisionalmente, se llega a la lista detallada de indicadores que aparece en el informe «Fichero de indicadores sociales sobre la situación de la mujer». ${ }^{2}$

En la tercera etapa se completan los indicadores de otras áreas y fuentes y se organiza la informatización de los indicadores.

El área de población y familia está siendo completada utilizando fundamentalmente la Encuesta de Fecundidad. El área de delitos y faltas ha sido investigada utilizando el Programa Estadístico de Seguridad del Ministerio del Interior. Además, se ha realizado una exploración de los ficheros de gestión de la Seguridad Social. ${ }^{3}$

Respecto a las áreas ya informatizadas, se encuentra grabada la información de los indicadores de empleo para el período 1982-87, y la mayor

2. Véase Moltó (1976).

3. Véase Moltó (1977 a) y (1977 b). 
parte de los indicadores de salud tienen ya los encabezamientos correspondientes. Ambas áreas se encuentran bajo la responsabilidad directa de la Subdirección de Estudios del Instituto de la Mujer. Las cintas que contienen la información del área de educación han sido grabadas por el CIDE.

\section{APLICACION DEL MODELO DE INDICADORES PARA EL ESTUDIO DE LA SITUACION DE LA MUJER EN ESPAÑA}

Como se ha destacado anteriormente, los indicadores sirven para cuantificar los principales rasgos de la situación de las mujeres en España.

A continuación se realiza un breve análisis de la situación, y se establecen comparaciones, tanto en el tiempo como en el espacio. Las comparaciones temporales serán diferentes dependiendo del indicador utilizado, ya que la evolución de aquellas características que varían rápidamente debe ser captada en un corto espacio de tiempo, en contraposición a las que experimentan variaciones en un largo plazo.

La comparación a nivel espacial, se establecerá, cuando sea posible, con los países de la CEE.

En definitiva, se trata de realizar un esbozo de la situación de la mujer en las diferentes áreas (demografía y familia, salud, educación y trabajo) en las que se ha dividido el análisis.

Con respecto a la dinámica demográfica, cabe destacar que el cambio que ha experimentado la población por sexo en España a lo largo del período 1970-1984 ha sido positivo, con una tasa de incremento anual acumulativo de 0,09 por 100 , tanto en el caso de las mujeres como en el de los varones. Éste ha sido superior al de la CEE, en la cual dicha tasa de crecimiento es 0,04 (cuadro 1 ).

En lo referente a la evolución reciente de la población por grupos de edad, cabe destacar que únicamente los varones y mujeres menores de 15 años tienen una participación porcentual en el total de la población ligetamente superior en España que en la CEE (cuadro 2). En todo caso, se observa que en el período 1970-1980, el número de varones nacidos por cada 1.000 mujeres nacidas ha aumentado a una tasa de crecimiento relativa de 1,8 por 100 en comparación con la CEE, que es de 0,2 por 100 .

En los cuadros 3 y 4 se observa que las tasas de natalidad y mortalidad en España en el período 1970-1980 han experimentado decrecimientos de 4,4 y 0,6 puntos, respectivamente, mientras que en la CEE dichas disminuciones son menores en ambos casos. A su vez, la tasa de crecimiento vegetativo ha sufrido una mayor reducción en España que en la $\mathrm{CEE}$. 
Sistema de indicadores sociales sobre la mujer

Así, el apreciable decrecimiento de la natalidad y el aumento de la esperanza de vida contribuyen al envejecimiento progresivo de la población. española. En el cuadro 5 se observa que la esperanza de vida, que continúa. siendo superior en las mujeres que en los varones, ha experimentado en España entre 1960 y 1970 incrementos superiores en la mujer que en el varón, y dichos incrementos son inferiores en la CEE.

\section{Cuadro 1}

Población media por sexo

\begin{tabular}{llrr}
\hline Ambito & Sexo & 1970 & 1984 \\
\hline España & Varones & $16.575,0$ & $18.852,5$ \\
& Mujeres & $17.204,0$ & $19.534,3$ \\
CEE-12 & Varones & $147.439,0$ & $156.249,0$ \\
& Mujeres & $155.949,0$ & $165.024,0$ \\
\hline
\end{tabular}

Fuente: EUROSTAT. Estadísticas demográficas.

\section{Cuadro 2}

Población por sexo y grupos de edad

(Estimación 1 de enero de 1986)

\begin{tabular}{crrrr}
\hline $\begin{array}{c}\text { Ambito } \\
y \text { grupos de edad }\end{array}$ & Varones & $\%$ & Mujeres & $\%$ \\
\hline España & & & & \\
$0-14$ & $4.525,1$ & 23,9 & $4.256,2$ & 21,7 \\
$15-44$ & $8.425,0$ & 44,5 & $8.246,7$ & 42,0 \\
$45-64$ & $4.097,0$ & 21,6 & $4.345,8$ & 22,1 \\
$65+$ & $1.886,5$ & 10,0 & $2.766,2$ & 14.1 \\
Total & $18.933,6$ & 100,0 & $19.614,9$ & 100,0 \\
\hline CEE-12 & & & & \\
$0-14$ & $32.948,3$ & 21,1 & $31.264,6$ & 18,9 \\
$15-44$ & $71.129,3$ & 45,5 & $69.261,2$ & 42,0 \\
$45-64$ & $35.495,4$ & 22,7 & $38.162,0$ & 23,1 \\
$65+$ & $16.675,2$ & 10,7 & $26.336,7$ & 15,9 \\
Total & $156.248,2$ & 100,0 & $165.024,5$ & 100,0 \\
\hline
\end{tabular}

Fuente: EUROSTAT. Estadísticas demográficas. 
El decrecimiento de la natalidad lleva aparejado un decrecimiento del índice de fecundidad o número medio de hijos por mujer, que ha pasado en España de 2,85 en 1970 a 2,19 en 1980 , y se ha operado también una disminución de la edad media de la madre en el nacimiento de los hijos, que en 1970 era de 29,5 años y en 1980 de $28,2 .^{4}$

De acuerdo con el cuadro 6 , las tasas de fecundidad según la edad de la madre que han experimentado un mayor descenso relativo, en el mismo período, son las correspondientes a los grupos de edad extremos. El descenso en el grupo de 15 a 19 años es de aproximadamente 80 por 100 y entre 45 y 49 años de 60 por 100 . La tasa de fecundidad de las mujeres entre 20 y 24 años únicamente ha experimentado una disminución de 4,2 por 100.

\section{CUADRo 3}

Tasas de natalidad

\begin{tabular}{|c|c|c|c|c|}
\hline & \multicolumn{2}{|c|}{1970} & \multicolumn{2}{|c|}{1980} \\
\hline & España & $C E E-12$ & España & $C E E-12$ \\
\hline Tasa de natalidad & 19,6 & 16,4 & 15,2 & 13,0 \\
\hline $\begin{array}{l}\text { Varones nacidos por } 1.000 \\
\text { mujeres nacidas }\end{array}$ & $1.059,0$ & $1.058,0$ & $1.078,0$ & $1.060,0$ \\
\hline $\begin{array}{l}\text { Nacimientos ilegítimos por } \\
1.000 \text { nacidos vivos }\end{array}$ & 13,6 & 47,7 & 39,2 & 73,8 \\
\hline $\begin{array}{l}\text { Nacidos muertos por } 1.000 \\
\text { nacidos vivos y muertos }\end{array}$ & 17,6 & 13,8 & 7,8 & 8,1 \\
\hline
\end{tabular}

Fuente: EUROSTAT. Estadísticas demográficas.

La tasa de matrimonios en este periodo ha experimentado también un descenso relativamente mayor en España que en la CEE, con tasas de decrecimiento relativas para el período considerado del 30 por 100 y 20 por 100 , respectivamente (cuadro 7). Adicionalmente, las tasas de natalidad denominada «ilegítima» ${ }^{5}$ han aumentado considerablemente entre 1970 y

4. Véase Memoria Estadística 1984-1985 del Ministerio de Sanidad y Consumo, Madrid, 1986.

5. Tras la reforma de filiación desaparece la distinción entre hijos legítimos e ilegítimos. Aunque dicha reforma es muy positiva a efectos de eliminar discriminaciones entre los hijos, sin embargo, es interesante disponer de estadísticas respecto al número de hijos de uniones libres, no matrimoniales. A este respecto, puede verse el estudio realizado en Francia por Deville y Naulleau (1982). 
Sistema de indicadores sociales sobre la mujer

1980. Partiendo de niveles considerablemente inferiores en España que en la $\mathrm{CEE}$, el crecimiento relativo experimentado durante esta década es extraordinariamente grande en España (con una tasa de crecimiento de 188,2 por 100 ), en comparación al de la CEE (con una tasa de crecimiento de 54,7 por 100) (cuadro 3 ).

\section{Cuadro 4}

Tasas de mortalidad

\begin{tabular}{|c|c|c|c|c|}
\hline & \multicolumn{2}{|c|}{1970} & \multicolumn{2}{|c|}{1980} \\
\hline & España & $C E E-12$ & España & $C E E-12$ \\
\hline Tasa de mortalidad & 8,3 & 10,6 & 7,7 & 10,3 \\
\hline $\begin{array}{l}\text { Muertes de menores de } \\
1 \text { año por } 1.000 \text { nacidos } \\
\text { (mortalidad infantil) }\end{array}$ & 28,1 & 24,1 & 12,3 & 12,4 \\
\hline $\begin{array}{l}\text { Muerte de menores de } 28 \\
\text { días por } 1.000 \text { nacidos vivos } \\
\text { Tasa de mortalidad }\end{array}$ & 17,5 & 16,1 & 8,5 & 7,1 \\
\hline perinatal & 25,5 & 25,3 & 8,5 & 14,0 \\
\hline
\end{tabular}

Fuente: EUROSTAT. Estadísticas demográficas. Ministerio de Sanidad y Consumo: Mernoria estadística 1984-1985.

Así, un fenómeno importante a tener en cuenta es la unión libre, que se generaliza progresivamente en los grupos sociales de nivel de instrucción superior. La cohabitación, que es difícilmente cuantificable mediante las estadísticas disponibles, ${ }^{6}$ tiene unas pautas de comportamiento con respecto a fenómenos tales como la fecundidad, la duración de la unión $\mathrm{y}$, en general, las actitudes ante la vida, diferentes de la unión por matrimonio.

Por otro lado, una característica sobresaliente respecto a la organización familiar es la relacionada con las familias monoparentales, ${ }^{7}$ por sus peculiares circunstancias en cuanto a la necesidad de servicios sociales. Conviene destacar, sin embargo, la multiplicidad de causas (viudedad, separación, divorcio, emigración, etc.) por su heterogeneidad.

La proporción de familias monoparentales en España es muy similar

6. Para analizar el intento de medición sobre el fenómeno de la cohabitación realizado en Francia, véase Audirac (1986).

7. Se entiende por familia monoparental la convivencia de un solo miembro de la pareja con hijos no emancipados. 
a la de los países de la CEE, según puede apreciarse en el cuadro 8. También conviene tener presente que un 7,2 por 100 del total de familias son monoparentales a cargo de la mujer, y sólo un 1,9 por 100 del total de familias son monoparentales a cargo del hombre. ${ }^{8}$ Esto puede constituir un indicador de la situación de precariedad de las familias monoparentales.

La separación y divorcio constituyen una fuente de alimentación de familias monoparentales $y$, en menor medida, de uniones libres. La población separada y divorciada sufre un notable incremento en la década de los setenta, y la tasa de crecimiento relativa es de aproximadamente 200 por 100. Aunque el hecho de la inexistencia de una ley de divorcio hasta 1981 relativiza estas cifras, que en el caso de la población de mujeres divorciadas asciende a 187,3 por 100 y a 221,7 por 100 en el caso de la población de varones divorciados (cuadro 9). ${ }^{9}$

En lo referente al ámbito de la salud ${ }^{10}$ cabe destacar, en primer lugar, con respecto a las condiciones de asistencia sanitaria, los riesgos asociados a embarazo y parto de la mujer y todo lo relacionado con la mortalidad infantil.

La tasa de mortalidad maternal ha descendido considerablemente entre 1975 y 1979 , pasando de 21,66 a 11,64 , lo que supone un ritmo de decrecimiento anual acumulativo de 14,4 por 100 .

$\mathrm{La}$ tasa de mortalidad perinatal ha experimentado un descenso superior en España que en la CEE, y el ritmo de decrecimiento anual acumulativo en el período $1970-1980$ es de 10,3 por 100 y de 5,7 por 100 , respectivamente. En cuanto a la tasa de mortalidad de menores de 28 días, las tasas de decrecimiento son de 7 por 100 y 7,9 por 100 en España y la CEE, respectivamente. Por último, la mortalidad infantil en España ha experimentado también un fuerte decrecimiento de 7,9 por 100 en comparación con 6,4 por 100 en la CEE (cuadro 4). Todo lo anterior indica la mejora relativa de los cuidados sanitarios en este periodo.

Respecto a las causas de muerte, hay que destacar que las mujeres tienen una tasa de mortalidad más alta que los varones en enfermedades de

8. Véase el Informe sobre la situación social de la mujer, diciembre de 1986, Instituto de la Mujer, Madrid, 1987.

9. Villac (1983) realiza una tipología de los hogares en Francia a partir de los Censos de Población de 1962, 1968 y 1975 y de las Encuestas sobre el Empleo de 1975 y 1981, relacionando las características de los hogares tipificados con la participación en la actividad económica.

10. Lo que sigue se encuentra basado, en parte, en el informe no publicado «Indicadores sociales sobre la situación de las mujeres. Área de Salud», elaborado por Concha Cifrián, Rosa Martínez, Carmen Martínez Ten y Sara Velasco. 
las glándulas endocrinas, del aparato circulatorio, de la piel, del sistema osteomuscular y signos y enfermedades mal definidos (cuadro 10). Hay que destacar que la mortalidad por cáncer de mama, que es la principal causa de muerte de las mujeres entre 45 y 64 años, ha experimentado un fuerte aumento en la última década. La tasa de crecimiento relativa, que en España es de 26,7 por 100 , se aproxima a la de los países de alto riesgo, como Inglaterra.

La incidencia de las enfermedades en la mujer se mide, entre ottos indicadores, por la tasa de morbilidad hospitalaria, que en 1983 resultó ser de 97,5 por 100 , en comparación con 85,2 por 100 en el caso de los varones. Analizándose las tasas de admisión hospitalaria por grupos de edad, se observa que, excepto en los grupos de edad que coinciden con el ciclo reproductivo (15 a 44 años), la tasa de admisión hospitalaria es mayor en los varones. Asimismo, la tasa global de utilización hospitalaria es 1,26 veces superior en los hombres que en las mujeres, siendo la estancia media más corta en las mujeres que en los hombres (del orden de cinco días menos).

\section{Cuadro 5}

Esperanza de vida

\begin{tabular}{|c|c|c|c|c|c|}
\hline \multirow[b]{2}{*}{ Sexo } & & \multicolumn{2}{|c|}{1970} & \multicolumn{2}{|c|}{1980} \\
\hline & & España & $C E E-12$ & España & $C E E-12$ \\
\hline \multicolumn{6}{|c|}{ Mujeres } \\
\hline & 0 años & 72,1 & 73,1 & 76,2 & 75,1 \\
\hline & 40 años & 36,8 & - & 38,6 & - \\
\hline \multicolumn{6}{|c|}{ Varones } \\
\hline & 0 años & 67,4 & 67,3 & 70,4 & 68,6 \\
\hline & 40 años & 33,1 & - & 33,8 & - \\
\hline
\end{tabular}

Fuente: EUROSTAT. Estadísticas demográficas.

Finalmente, los indicadores clásicos en el ámbito de la salud son indicadores negativos, basados en el concepto de salud como ausencia de enfermedad. La OCDE ha propuesto a este respecto la elaboración de indicadores positivos que reflejen la calidad de vida de las personas. La calidad de vida se encuentra intimamente relacionada con las condiciones de vida y vivienda en general, y en particular con el nivel educativo y cultural, las condiciones laborales, etc. 
«Papers»: Revista de Sociologia

\section{CuAdro 6}

Tasas de fecundidad según edad de la madre

\begin{tabular}{lrrc}
\hline Grupo de edad & 1970 & 1980 & $\begin{array}{c}\text { Tasa } \\
\text { variación } \\
\times 100\end{array}$ \\
\hline De 15 a 19 años & 14,1 & 25,3 & 70,4 \\
De 20 a 24 años & 121,0 & 115,9 & 4,2 \\
De 25 a 29 años & 197,2 & 148,1 & 24,9 \\
De 30 a 34 años & 131,4 & 92,1 & 29,9 \\
De 35 a 39 años & 76,8 & 45,4 & 40,9 \\
De 40 a 44 años & 26,0 & 14,8 & 43,1 \\
De 45 a 49 años & 3,2 & 1,3 & 59,4 \\
\hline
\end{tabular}

Fuente: INE. Movimiento Natural de la Población Española.

Los rasgos básicos de la situación de la mujer en el área de educación hacen referencia a la participación de las mujeres en el alumnado, en el cuerpo docente y en el analfabetismo adulto.

\section{CuAdro 7}

Matrimonios

\begin{tabular}{ccccccc}
\hline & \multicolumn{2}{c}{1970} & & \multicolumn{2}{c}{1980} \\
\cline { 2 - 3 } \cline { 5 - 6 } \cline { 5 - 6 } & España & CEE-12 & & España & CEE-12 \\
\hline Tasa matrimonios & 7,3 & 7,8 & & 5,7 & 6,2 \\
\hline
\end{tabular}

Fuente: EUROSTAT. Estadísticas demográficas.

La participación femenina en el alumnado del sistema educativo es muy similar a la masculina en todos los niveles, con algunas diferencias de tipo cualitativo. Entre estas diferencias cabe destacar una mejor adaptación de las niñas en los niveles de preescolar y EGB, aunque ello no implica un mejor desarrollo del potencial intelectual, a juzgar por los resultados de las pruebas de rendimiento y aptitudes, la desigual distribución por ramas en la enseñanza media, etc. Hay que destacar también el fuerte incremento del 33 por 100 del alumnado femenino en la universidad en el último quinquenio, frente al incremento del 12 por 100 de 
los varones y la tendencia hacia un aumento, aunque mucho más lento, en la participación de la mujer en los estudios técnicos superiores.

\section{Cuadro 8}

Proporción de familias monoparentales en diversos países

\begin{tabular}{lr}
\hline Bélgica (1970) & 9,3 \\
Dinamarca (1980) & 12,0 \\
Francia (1980) & 10,1 \\
República Federal de Alemania (1982) & 11,4 \\
Irlanda (1975) & 9,4 \\
Gran Bretaña (1971) & 10,0 \\
España (1975) & 9,1 \\
\hline
\end{tabular}

Fuente: Datos tomados de Situación Social de la Mujer en España.

\section{Cuadro 9}

Población separada y divorciada

\begin{tabular}{lrrr}
\hline & Total & Varones & \multicolumn{1}{c}{ Mujeres } \\
\hline 1950 & $2,1(0,1 \%)$ & 0,5 & $1,6(0,1 \%)$ \\
1960 & $25,6(0,8 \%)$ & $8,2(0,6 \%)$ & $17,4(1,1 \%)$ \\
1970 & $81,5(2,4 \%)$ & $29,5(1,8 \%)$ & $52,0(3,0 \%)$ \\
1981 & $244,3(6,5 \%)$ & $94,9(5,1 \%)$ & $149,4(7,8 \%)$ \\
\hline
\end{tabular}

Fuente: INE. Anuario estadístico de España.

Cuadro 10

Tasas de mortalidad por algunas causas seleccionadas en España en 1980

\begin{tabular}{lrrc}
\hline & Mujeres & Varones & Ratio $M / V$ \\
\hline Enfermedades de glándulas endógenas & 26,45 & 15,08 & 1,75 \\
Enfermedades del aparato circulatorio & 365,18 & 338,14 & 1,07 \\
Enfermedades de la piel & 0,62 & 0,34 & 1,82 \\
Enfermedades del sistema osteomuscular & 1,97 & 1,05 & 1,87 \\
Signos y enfermedades mal definidas & 30,23 & 23,04 & 1,31 \\
\hline
\end{tabular}

Fuente: Informe sobre la Situacion de las Mujeres: Area de Salud. 
La participación de la mujer como docente registra grandes diferencias entre los distintos niveles, pues se observa una mayor proporción de profesorado femenino a menor edad del alumnado y en educación especial (cuadro 11).

Mención especial merece el analfabetismo de adultos, ya que, tal como pone de relieve la OCDE, éste es un buen indicador del estado de la educación y cultura de un país. Así, la tasa de mujeres analfabetas en 1981 era del 8,9 por 100 , superior a la tasa de varones analfabetos $(3,6$ por 100), aumentando con la edad, en mayor medida relativamente, en el caso de las mujeres que en el de los varones. La tasa de analfabetismo de adultos aumenta con la edad, correspondiendo la máxima tasa en ambos casos al grupo de edad superior a los 65 años, y dicha tasa en las mujeres se sitúa 18,8 puntos porcentuales por encima de su media y sólo 8,6 puntos porcentuales en el caso de los hombres. Por último, si consideramos las estimaciones sobre analfabetismo funcional, las tasas tanto para varones como para mujeres sufren incrementos considerables, pues se sitúan entre el 20 y el 30 por 100 (cuadro 12).

Respecto a la actividad laboral de la mujer en España, cabe destacar el crecimiento experimentado por las tasas de actividad de las mujeres no solteras en edad reproductiva, especialmente en el caso de las mujeres entre 25 y 29 años, cuyo ritmo anual de crecimiento es del 7,3 por 100 en el periodo 1976-1985 (cuadro 13).

La incorporación de la mujer al mercado de trabajo se puede considerar irreversible, tanto en España como en los países de la CEE. Según Huet (1982), la incorporación de la mujer al trabajo en Francia se produce, por un lado, gracias a un cambio de actitud ante el trabajo de las propias mujeres; y por otro lado, debido al aumento de las posibilidades de empleo merced a una expansión de la actividad en el sector terciario y el desarrollo de formas de empleo más flexibles (a tiempo parcial) y también menos estables, sobre todo en el sector informal.

Otro rasgo esencial es la concentración del empleo femenino en un reducido número de ramas de actividad, con la consiguiente feminización de dichas ramas. Así, el 63,5 por 100 de todas las mujeres ocupadas están concentradas en sólo cinco ramas de actividad que, por orden de importancia, son las siguientes: comercio, agricultura, servicios personales y domésticos, educación y sanidad.

La segregación ocupacional se mide mediante el índice de disimilaridad que indica el grado de integración de varones y mujeres en las diferentes ramas de actividad, que es independiente del tamaño relativo de la población activa ocupada, tanto masculina como femenina. Los valores del índice de disimilaridad, que oscila entre cero -que indica distribución 
igualitaria- $-\mathrm{y}$ cien —que indica segregación total-, han experimentado un descenso relativo del 4,5 por 100 , en el período 1976-1985, pasando de 48,18 al principio del periodo a 46,00 al final del mismo. Ello indica que en 1976 aproximadamente la mitad de las mujeres debían cambiar de ocupación para eliminar la segregación ocupacional. ${ }^{11} \mathrm{El}$ índice de disimilaridad, calculado en base a la misma fórmula en Francia a partir de los datos de la Encuesta sobre la Estructura del Empleo, asciende en 1979 a 35,00 (véase Huet, 1983). Aunque las comparaciones internacionales deben interpretarse con precaución, puesto que dicho índice será diferente según la desagregación de ocupaciones (en España los datos utilizados para el cálculo de este índice han sido los correspondientes a las 62 ocupaciones de la EPA), se observa que el indice de desigualdad es superior en España que en Francia.

Por otra parte, la incidencia del desempleo en España es mayor en el caso de las mujeres, cuya tasa de desempleo asciende a 25,5 por 100 en el último trimestre de 1985, que en el caso de los varones, con tasa de desempleo de 20,4 por 100 , según la EPA. Las tasas de desempleo de las mujeres solteras son considerablemente superiores a las de las no solteras; sin embargo, han crecido a un ritmo inferior. Así, la tasa de desempleo de las mujeres solteras ha experimentado en el período 1976-1985 un crecimiento anual en media de 18,7 por 100 , pasando de 8,6 por 100 en 1976 a 40,3 por 100 en 1985 , en comparación con las mujeres no solteras cuya tasa de paro ha crecido a un ritmo medio anual de 25,8 por 100 , pasando de 1,8 por 100 al principio del período a 14,2 por 100 al final del mismo.

Paralelamente, las demandas de empleo registradas en las Oficinas de Empleo han experimentado un crecimiento medio anual de 17,6 por 100 en el caso de las mujeres, pasando el número de demandas de empleo de 554,3 en 1978 a $1.689,9$ en 1985 . El crecimiento en el caso de los varones ha sido de 8,6 por 100 en el período considerado, pasando de $1.877,4$ en 1978 a $3.346,3$ en 1985 . El total de colocaciones registradas ha crecido a una tasa anual acumulativa de 15,4 por 100 en el caso de las mujeres, pasando de 243,2 en 1978 a 664,5 en 1985 . Mientras que en el caso de los varones dicho crecimiento ha sido inferior, con una tasa de 8,6 por 100 en media anual.

11. Un estudio en profundidad sobre este tema y los datos estadísticos correspondientes se encuentra en el Informe sobre la situación social de la mujer en España, diciembre de 1986. Madrid, 1987.

6. 
«Papers»: Revista de Sociologia

\section{Cuadro 11}

Número y proporción de mujeres docentes por niveles de enseñanza

\begin{tabular}{lrc}
\hline Nivel & Mujeres & $\begin{array}{r}\text { \% mujeres } \\
\text { sobre total }\end{array}$ \\
\hline Preescolar & 36.351 & 95,4 \\
Enseñanza General Básica & 131.478 & 60,0 \\
Bachillerato y COU & 34.143 & 47,9 \\
Formación Profesional & 14.502 & 32,0 \\
E. Segundo Grado & 2.350 & 38,0 \\
E. Permanente de Adultos & 1.359 & 43,7 \\
E. Especial & 7.358 & 73,0 \\
Escuelas Universitarias & 3.787 & 30,5 \\
Facultades y Colegios Universitarios & 6.718 & 25,3 \\
E. Técnicas Superiores & 498 & 9,4 \\
E. Tercer Grado Superior & 543 & 22,1 \\
\multicolumn{1}{c}{ Total } & 239.087 & 54,4 \\
\hline
\end{tabular}

Fuente: INE. Estadística de la Enseñanza en España. Curso 83-84.

El impacto de la formación sobre el desarrollo profesional de las mujeres ha sido puesto de manifiesto en Francia por Strecker-Seeborg y otros (1984). Un análisis de los datos de los alumnos que han terminado sus estudios en los programas de formación del INEM en 1985 muestra que la distribución de varones según familias profesionales es más uniforme que la de las mujeres. El 72,3 por 100 de las mujeres se concentran en seis ramas, que son, por orden de importancia, las siguientes: Administración y oficinas $(18,9$ por 100$)$, textil ( 14,2 por 100$)$, sanitaria $(11,2$ por $100)$, peluquería y estética $(10,1$ por 100$)$, técnicos empresariales $(9,6$ por 100$)$ e informática (8,3 por 100).

Por último, hay que destacar que las condiciones de vida y trabajo en las mujeres se encuentran íntimamente relacionadas. Sin embargo, la interrelación entre la disminución de las tasas de fecundidad y el aumento de la participación de la mujer en el mercado de trabajo no tiene una interpretación inmediata. ${ }^{12} \mathrm{La}$ socialización temprana de los hijos fuera del hogar, la simplificación de las tareas domésticas y su redistribución entre

12. Véase la interpretación de Lery (1984) en Francia. 
los miembros de la familia, ${ }^{13} \mathrm{y}$ en definitiva la distribución del tiempo, que disminuye el tiempo de ocio de las mujeres con responsabilidades domésticas con el consiguiente aumento del riesgo de enfermar, son elementos que pueden servir para matizar este tipo de conclusiones. Como afirma Roy (1982) al hablar de la distribución del tiempo de padres y madres de familia numerosa, las madres de familia activas son las que menos ocio tienen, cualquiera que sea el número de hijos.

\section{CuAdro 12}

Personas de 10 y más años que se declaran analfabetas

\begin{tabular}{lrrrrrr}
\hline Edad & Todos & $\%$ & Hombres & $\%$ & Mujeres & $\%$ \\
\hline \multicolumn{1}{c}{ Total } & 1.991 .581 & 6,36 & 551.818 & 3,6 & 1.439 .763 & 8,9 \\
10 a 14 años & 19.886 & 0,6 & 10.337 & 0,6 & 9.549 & 0,6 \\
15 a 19 años & 27.377 & 0,8 & 14.280 & 0,9 & 13.097 & 0,8 \\
20 a 24 años & 32.069 & 1,1 & 14.652 & 1,0 & 17.417 & 1,2 \\
25 a 34 años & 86.653 & 1,7 & 32.884 & 1,3 & 53.769 & 2,2 \\
35 a 44 años & 187.373 & 4,4 & 54.891 & 2,6 & 132.482 & 6,1 \\
45 a 54 años & 363.885 & 7,9 & 110.240 & 4,8 & 253.645 & 10,8 \\
55 a 64 años & 368.460 & 10,1 & 104.050 & 6,1 & 264.410 & 13,7 \\
65 años y más & 905.878 & 21,4 & 210.483 & 12,2 & 695.395 & 27,7 \\
\cline { 2 - 8 } Total 15 o más & 1.971 .695 & 7,04 & 541.481 & 4,01 & 1.430 .214 & 9,9 \\
Total 15-64 años 1.065 .817 & 4,5 & 330.998 & 2,8 & 743.819 & 6,1 \\
\hline Analfabeto funcional & & & 22,54 & & 29,0 \\
\hline
\end{tabular}

Fuente: INE. Censo de 1981.

13. Un estudio sobre la organización doméstica en Francia puede verse en Glaude y Singly (1986). 
«Papers»: Revista de Sociologia

CuAdro 13

Tasas de actividad por sexo, estado civil y grupos de edad

\begin{tabular}{cccccc}
\hline & \multicolumn{2}{c}{1976} & & \multicolumn{2}{c}{1985} \\
\cline { 2 - 3 } \cline { 5 - 6 } Mujeres & Solteras & No solteras & & Solteras & No solteras \\
\hline Total & 56,2 & 18,7 & & 49,5 & 20,7 \\
16 a 19 años & 40,0 & 31,5 & & 31,8 & 30,6 \\
20 a 24 años & 68,7 & 25,8 & & 59,8 & 39,7 \\
25 a 29 años & 76,6 & 22,0 & & 81,2 & 41,4 \\
30 a 34 años & 76,2 & 20,6 & & 79,2 & 35,1 \\
35 a 39 años & 70,6 & 22,9 & & 79,8 & 29,2 \\
40 a 44 años & 68,3 & 24,7 & & 71,0 & 27,2 \\
45 a 49 años & 67,0 & 24,2 & & 71,0 & 24,3 \\
50 a 54 años & 60,6 & 23,6 & & 64,6 & 21,4 \\
55 a 59 años & 53,9 & 20,5 & & 63,7 & 20,0 \\
60 a 64 años & 46,5 & 17,7 & & 42,3 & 13,5 \\
65 a 69 años & 27,5 & 9,5 & & 7,0 & 4,3 \\
70 años y más & 8,4 & 3,3 & & 2,4 & 0,9 \\
\hline
\end{tabular}

Fuente: EPA.

\section{CONCLUSIONES}

Es indudable la importancia que tiene un sistema de indicadores sociales sobre las mujeres, tanto a efectos de conocimiento de su situación como en la evaluación de los resultados de las políticas públicas. Sin embargo, no menos relevante es su contribución al proceso de conceptualización y a la señalización de las principales dimensiones de cada una de las áreas en las que se ha dividido la elaboración del sistema de indicadores por razones puramente prácticas. Es importante que en el proceso de construcción se redefinan constantemente los problemas más relevantes.

Se trata de realizar, como afirma Land (1975), un análisis del ciclo vital de las personas - y en nuestro caso, de las mujeres en relación con los hombres- en términos de su espacio vital, tanto en lo referente a las condiciones objetivas como en las valoraciones y bienestar subjetivo. Ello implica la ampliación de la lista básica de indicadores, mediante estudios 
especificos sobre actitudes, tarea que el Instituto de la Mujer ha comenzado ya a realizar. ${ }^{14}$

Finalmente, por lo que respecta a la implementación del sistema de indicadores de la ONU en España, los resultados obtenidos hasta el momento son satisfactorios, si bien es necesario completar dicho modelo, explorando en mayor profundidad las fuentes de información ya utilizadas, otras fuentes disponibles y definiendo nuevas áreas de preocupación social. También alrededor de estas cuestiones el Instituto de la Mujer tiene establecido un plan de trabajo en la actualidad.

14. Miranda (1987) recoge en su trabajo los estudios sociológicos financiados per el Instituto de la Mujer entre 1983 y 1986, scbre temas tan diversos como el feminismo, la política, el trabajo, los malos tratos, la publicidad, las tareas domésticas, etc. También se han realizado análisis sobre las actitudes de los varones. Véase a este respecto Alberdi y otras (1987) e INNER (1987). Las desigualdades en el uso del tiempo constituyen también objeto de investigación. Véase Izquierdo, Del Río y Rodríguez (1987). 


\section{BIBLIOGRAFIA}

Alberdi, I. y otras (1987): «Actitudes de los varones ante el cambio familiar», Instituto de la Mujer. Informe no publicado.

Astelarra, J. (1986): «Indicadores sociales sobre la situación de las mujeres. Primer informe sobre el proyecto de investigación: Sistema de indicadores sociales sobre la situación de la mujer en España», Instituto de la Mujer, Barcelona, febrero. Informe no publicado.

Audirac, P. A. (1986): «La Cohabitation: Un milion de couples non mariés», Economie et Statistique, 185, pp. 13-33.

Cifrian, C. y otras (1987): «Indicadores sociales sobre la situación de las mujeres: Ârea de Salud», Instituto de la Mujer. Informe no publicado.

Deville, J. C., y Naulleau, E. (1982): «Les Nouveaux Enfants Naturels et Leurs Parents», Économie et Statistique, 145, pp. 61-81.

Glaude, M. y Singly, F. (1986): «L'Organisation Domestique: Pouvoir et Négotiation», Economie et Statistique, 187, pp. 3-30.

Hernández Iglesias, F. (1987): «Familias monoparentales en España», Instituto de la Mujer, Ministerio de Cultura. Informe no publicado.

Horn, R. V. (1980): «Social Indicators: Meaning, Methods and Applications», International Journal of Social Economics, 7 (8), pp. 421-460.

Huet, M. (1982): «La progression de l'activité féminine, est-elle irréversible?», Economie et Statistique, 145, pp. 3-17.

Huet, M. (1983): «La concentration des emplois féminins», Economie et Statistique, 154, pp. 33-46.

Inner (1987): "Actitudes de los varones ante el proceso de emancipación de las mujeres», Instituto de la Mujer. Informe no publicado.

Izquierdo, M. J.; Del Río, O y Rodríguez, A. (1987): «La expresión de la desigualdad social de las mujeres en el uso y el paso del tiempo. Parte I: El uso del tiempo», Instituto de la Mujer. Informe no publicado.

Land, K. C. (1975): «Theories, Models and Indicators of Social Change», International Social Science Journal, 1.

Lery, A. (1984): «Les actives de 1982 n'ont pas moins d'enfants que celles de 1968», Economie et Statistique, 171-172, pp. 25-34. 
Miranda, M. J. (1987): Crónicas del desconcierto. Serie Estudios 8, Instituto de la Mujer, Ministerio de Cultura.

Moltó, M. L. (1986): «Fichero de indicadores sociales sobre la situación de la mujer», Instituto de la Mujer. Informe no publicado.

Moltó, M. L. (1987 a): «Indicadores sociales sobre la situación de la mujer. Explotación del programa estadístico de seguridad del Ministerio del Intetior», Instituto de la Mujer. Informe no publicado.

Moltó, M. L. (1987 b): «Exploración de los ficheros informatizados de la Seguridad Social», Instituto de la Mujer. Informe no publicado.

Ministerio de Cultura. Instituto de la Mujer (1987): Situación social de la mujer en España, Instituto de la Mujer, Ministerio de Cultura, diciembre, 1986.

ONU (1984): «Compiling Social Indicators on the Situation of Women. Studies in Methods», Series F. 32, United Nations, New York.

ONU (1984): «Improving Concepts and Methods for Statistics and Indicators on tre Situation of Women», Series F. 33, United Nations, New York.

Roy, C. (1982): «L'Emploi du Temps des Mères et Pères de Famille. Nombreuse», Économie et Statistiquie, 141 pp. 59-68.

Ruggles, R. y Ruggles, N. D. (1974): Social Indicators and a Framework for Social and Economic Accounts. American Statistical Association, ed.: Proceedings of the Social Statistics Section 1974, Washington D.C.

Streker-Seeborg, I. et al. (1984): «The Impact of Nontraditional Training on the Occupational Attainment of Women», Journal of Human Resources, XIX (4), pp. 452-471.

Torns, T. y Carrasquer, P. (1985): «Indicadores sociales sobre la situación de la mujer. Las propuestas de la ONU», Ponencia presentada al Seminario: Indicadores sociales sobre la situación de las mujeres, Barcelona, 12 al 14 de diciembre de 1985.

Villac, M. (1983): «Les structures familiales se transforment profondément», Économie et Statistique, 152, pp. 39-53. 